\title{
Photoproduction of pentaquark cascades from nucleons
}

\author{
W. Liu and C. M. Ko \\ Cyclotron Institute and Physics Department, Texas A\&M University, College Station, Texas 77843-3366, USA
}

(Received 6 January 2004; published 30 April 2004)

\begin{abstract}
The cross sections for production of the pentaquark baryon $\Xi_{5}^{+}$from the reaction $\gamma p \rightarrow K^{0} K^{0} \Xi_{5}^{+}$and of $\Xi_{5}^{--}$ from the reaction $\gamma n \rightarrow K^{+} K^{+} \Xi_{5}^{--}$are evaluated in a hadronic model that includes their couplings to both $\Sigma \bar{K}$ and $\Sigma \bar{K}^{*}$. Values for these coupling constants are determined from the empirical $\pi N N(1710)$ and $\rho N N(1710)$ coupling constants by assuming that $\Xi_{5}^{+}, \Xi_{5}^{--}$, and $N(1710)$ belong to the same antidecuplet of spin $1 / 2$ and positive parity as the pentaquark $\Theta^{+}$. Introducing empirical form factors at strong interaction vertices, we obtain a cross section of about $0.03-0.6 \mathrm{nb}$ for the reaction $\gamma p \rightarrow K^{0} K^{0} \Xi_{5}^{+}$and about $0.1-0.6 \mathrm{nb}$ for the reaction $\gamma n \rightarrow K^{+} K^{+} \Xi_{5}^{--}$at photon energy $E_{\gamma}=4.5 \mathrm{GeV}$, depending on the value of the coupling constant $g_{K^{*} \Sigma \Xi_{5}}$.
\end{abstract}

DOI: 10.1103/PhysRevC.69.045204

PACS number(s): 13.60.Rj, 14.20.-c, 24.85.+p

\section{INTRODUCTION}

The observation of the pentaquark $\Theta^{+}(u u d d \bar{s})$ baryon [1] in nuclear reactions induced by photons [2-5] and kaons [6] has prompted extensive theoretical studies on both its properties [7-22] as well as production [23-30] and decay mechanisms [31,32]. Although most models predict that $\Theta^{+}$ has spin $1 / 2$ and isospin 0 , their predictions on its parity vary widely. While the soliton model gives a positive parity and the lattice QCD study favors a negative parity, the quark model can give either positive or negative parities, depending on whether quarks are correlated or not. The parity of $\Theta^{+}$ affects both the magnitude of its production cross section in these reactions and the photon asymmetry in photonucleon reactions. With a positive $\Theta^{+}$parity, the production cross section is almost an order of magnitude larger than that for a negative parity $\Theta^{+}$as a result of a smaller $K N \Theta$ coupling constant in the latter case [25-27]. The positive parity also leads to a large positive photon asymmetry, which becomes negative if the $\Theta^{+}$parity is negative $[28,30]$.

There are other pentaquark baryons $\Xi_{5}^{+}$(uuss $\bar{d}$ ) and $\Xi_{5}^{--}$ $(d d s s \bar{u})$ in the same antidecuplet as $\Theta^{+}$. Although the $\Xi_{5}^{--}$ has already been observed recently in $p+p$ collisions at center-of-mass energy $\sqrt{s}=17.2 \mathrm{GeV}$ by NA49 Collaboration [33], its production in photonucleon reactions has not been studied. In this paper, we shall use the same hadronic model that was introduced for studying $\Theta^{+}$production in photonucleon reactions [23-25] to study the production of $\Xi_{5}^{+}$and $\Xi_{5}^{--}$in these reactions.

This paper is organized as follows. In Sec. II, we consider the reactions $\gamma p \rightarrow K^{0} K^{0} \Xi_{5}^{+}$and $\gamma n \rightarrow K^{+} K^{+} \Xi_{5}^{--}$for pentaquark cascade production, introduce the interaction Lagrangians needed for evaluating their cross sections, and discuss the coupling constants and form factors at strong interaction vertices. Results on the cross sections for these reactions are then shown and discussed in Sec. III. In Sec. IV, a brief summary is given. Finally, the formula for evaluating the cross section for reactions involving two particles in the initial state and three particles in the final state is derived in the Appendix.

\section{PENTAQUARK CASCADE PRODUCTION IN PHOTON-NUCLEON REACTIONS}

Possible reactions for pentaquark $\Xi_{5}$ production in photon-nucleon reactions near threshold are $\gamma p \rightarrow K^{0} K^{0} \Xi_{5}^{+}$ and $\gamma n \rightarrow K^{+} K^{+} \Xi_{5}^{--}$. To evaluate their cross sections, we use the hadronic model introduced in Refs. [23-25] for studying $\Theta^{+}$production in photon-nucleon reactions. This model is a generalization of the $\mathrm{SU}(3)$ flavor-invariant Lagrangian for the interactions between octet pseudoscalar mesons and baryons [34] to include the interactions of $\Theta^{+}$with nucleons and $K$ as well as $K^{*}$. Photon in this model is introduced as $\mathrm{U}_{\mathrm{em}}(1)$ gauge particle, and the symmetry breaking effects are taken into account phenomenologically by using empirical hadron masses and coupling constants. In the present study, we extend the model to include interactions of the pentaquark $\Xi_{5}$ with $\Sigma$ and $K$ or $K^{*}$. Since only three-point interactions appear in the interaction Lagrangians, relevant Feynman diagrams that contribute to these reactions are those shown in Figs. 1 and 2.

\section{A. Interaction Lagrangians}

The interaction Lagrangians relevant for evaluating the amplitudes shown in Figs. 1 and 2 are:

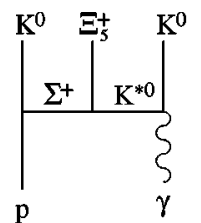

(1a)

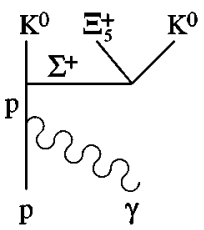

(1d)

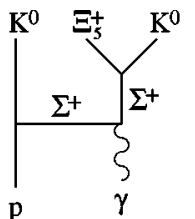

(1b)

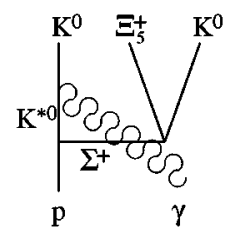

(1e)
FIG. 1. Diagrams for pentaquark $\Xi_{5}^{+}$production from the reaction $\gamma p \rightarrow K^{0} K^{0} \Xi_{5}^{+}$. 


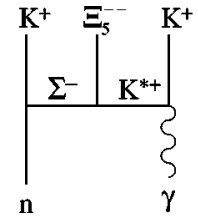

(2a)

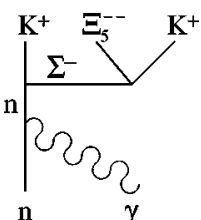

(2e)

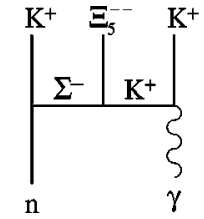

(2b)

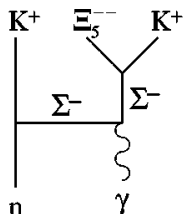

(2c)

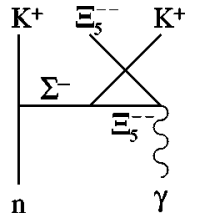

(2d)

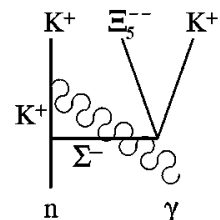

(2f)

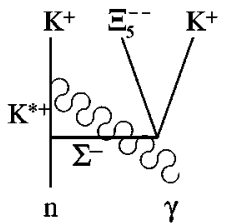

(2g)

$$
\begin{gathered}
\mathcal{L}_{K N \Sigma}=i g_{K N \Sigma} \bar{N} \gamma_{5} \vec{\Sigma} \cdot \vec{\tau} K+\text { H.c. } \\
\mathcal{L}_{K \Sigma \Xi_{5}}=i g_{K \Sigma \Xi} \bar{\Xi}_{5} \gamma_{5} \vec{\Sigma} \cdot \vec{T} K+\text { H.c. } \\
\mathcal{L}_{K^{*} N \Sigma}=g_{K^{*} N \Sigma} \bar{N} \gamma_{\mu} \vec{\Sigma} \cdot \vec{\tau} K^{* \mu}+\text { H.c. } \\
\mathcal{L}_{K^{*} \Sigma \Xi_{5}}=g_{K^{*} \Sigma \Xi \Xi_{5}} \gamma_{\mu} \vec{\Sigma} \cdot \vec{T} K^{* \mu}+\text { H.c. } \\
\mathcal{L}_{\gamma N N}=-e \bar{N}\left\{\gamma_{\mu} \frac{1+\tau_{3}}{2} A^{\mu}\right. \\
\left.-\frac{1}{4 m_{N}}\left[\kappa_{p}+\kappa_{n}+\tau_{3}\left(\kappa_{p}-\kappa_{n}\right)\right] \sigma_{\mu \nu} \partial^{\nu} A^{\mu}\right\} N, \\
\mathcal{L}_{\gamma \Sigma \Sigma}=-e \bar{\Sigma} \gamma_{\mu} Q_{1} \Sigma A^{\mu}, \\
\mathcal{L}_{\gamma K K}=i e\left[\bar{K} Q_{2} \partial K-\left(\partial_{\mu} \bar{K}\right) Q_{2} K\right] A^{\mu}, \\
\mathcal{L}_{\gamma K K^{*}}=g_{\gamma K K^{*}} \epsilon_{\alpha \beta \mu \nu} \partial^{\alpha} A^{\beta}\left[\left(\partial^{\mu} \bar{K}^{* \nu}\right) K+\bar{K} \partial^{\mu} K^{*} \nu\right] .
\end{gathered}
$$

In the above, $N, \Sigma$, and $\Xi_{5}$ denote, respectively, the nucleon isospin doublet, the $\Sigma$ isospin triplet, and the pentaquark $\Xi_{5}$ isospin quartet; $K$ and $K^{*}$ are the pseudoscalar and vector kaon isospin doublets, respectively; and $A_{\mu}$ denotes the photon. The Pauli matrices are given by $\vec{\tau}$, while $\vec{T}$ is an isospin transition operator represented by a $4 \times 2$ matrix with matrix elements given by $\left\langle\frac{3}{2} m\left|T_{\lambda}\right| \frac{1}{2} n\right\rangle=\left(\frac{3}{2} m \mid 1 \lambda \frac{1}{2} n\right)$, where $\lambda$ $=0, \pm 1$. The operators $Q_{1}=\operatorname{diag}(1,0,-1)$ and $Q_{2}$ $=\operatorname{diag}(1,0)$ are diagonal charge operators and $\epsilon_{\alpha \beta \mu \nu}$ denotes the antisymmetric tensor with the usual convention $\epsilon_{0123}=1$.

For the coupling constants $g_{K N \Sigma}$ and $g_{K^{*} N \Sigma}$, their values can be related to $g_{\pi N N}$ and $g_{\rho N N}$, which have empirical values of $g_{\pi N N}=13.5$ [35] and $g_{\rho N N}=3.25$ [36], by SU(3) symmetry, i.e., $g_{K N \Sigma}=(1-2 \alpha) g_{\pi N N}=-3.78$ and $g_{K^{*} N \Sigma}=g_{\rho N N}=3.25$ if we use the ratio $\alpha=D /(D+F)=0.64$ [37] for the $D$ - and $F$ - type interaction Lagrangians between pseudoscalar mesons and baryons. Similarly, we have $g_{K \Sigma \Xi_{5}}=g_{K N \Theta} / \sqrt{2}=2.16$ and $g_{K^{*} \Sigma \Xi_{5}}=g_{K^{*} N \Theta} / \sqrt{2}=1.27$ [38], where the values for $g_{K N \Theta}$ $=3.06$ and $g_{K^{*} N \Theta}=1.8$ have been determined in Ref. [25]
FIG. 2. Diagrams for pentaquark $\Xi_{5}^{--}$production from the reaction $\gamma n \rightarrow K^{+} K^{+} \Xi_{5}^{--}$. from the empirical $\pi N N(1710)$ and $\rho N N(1710)$ coupling constants using the SU(3) symmetry. Since the sign of $g_{K^{*} N \Theta}$ relative to $g_{K N \Theta}$ and $g_{K N \Sigma}$ is not fixed by the SU(3) symmetry, we shall consider both signs for the coupling constant $g_{K^{*} \Sigma \Xi_{5}}= \pm 1.27$ as well as the case of $g_{K^{*} \Sigma \Xi_{5}}=0$.

For photon coupling to nucleon, we include also its interaction with the anomalous magnetic moment of nucleons with empirical values of $\kappa_{p}=1.79$ and $\kappa_{n}=-1.91$. Since the anomalous magnetic moment of $\Theta^{+}$is not known, we neglect its coupling to photon.

The coupling constant $g_{\gamma K K^{*}}$ denotes the photon anomalous parity interaction with kaons and has the dimension of inverse of energy. Its value is $g_{\gamma K^{0} K^{*} 0}=0.388 \mathrm{GeV}^{-1}$ and $g_{\gamma K^{ \pm} K^{* \pm}}=0.254 \mathrm{GeV}^{-1}$ [24] using the decay width $\Gamma_{K^{* 0} \rightarrow K^{0} \gamma}$ $=0.117 \mathrm{MeV}$ of $K^{* 0}$ and $\Gamma_{K^{* \pm} \rightarrow K^{ \pm} \gamma}=0.05 \mathrm{MeV}$ of $K^{* \pm}$ to kaon and photon [39]. Although the sign of $g_{\gamma K K^{*}}$ relative to other coupling constants in the interaction Lagrangians is not known either, it is not relevant for our study as both constructive and destructive interferences among the diagrams in Fig. 1 and 2 are automatically taken into account by using different signs for the coupling constant $g_{K^{*} \Sigma \Xi_{5}}$.

\section{B. Amplitudes for the reactions $\gamma p \rightarrow K^{0} K^{0} \Xi_{5}^{+}$and $\gamma n \rightarrow K^{+} K^{+} \Xi_{5}^{--}$}

With the above interaction Lagrangians, we can write the amplitudes for the five diagrams in Fig. 1 as

$$
\begin{aligned}
\mathcal{M}_{1 a}= & 2 i g_{\gamma K^{0} K^{*} 0} g_{K^{*} \Sigma \Xi_{5}} g_{K N \Sigma} \frac{1}{\left[\left(p_{2}-p_{5}\right)^{2}-m_{K^{*}}^{2}\right]\left(t-m_{\Sigma}^{2}\right)} \\
& \times \epsilon_{\alpha \beta \mu \nu} p_{2}^{\alpha} p_{5}^{\beta} \epsilon^{\mu} \bar{\Xi}_{5}\left(p_{4}\right) \gamma^{\nu}\left(p_{1}-p_{3}+m_{\Sigma}\right) \gamma_{5} p\left(p_{1}\right), \\
\mathcal{M}_{1 b}= & -2 e g_{K \Sigma \Xi_{5}} g_{K N \Sigma} \frac{1}{\left(s_{1}-m_{\Sigma}^{2}\right)\left(t-m_{\Sigma}^{2}\right)} \\
& \times \bar{\Xi}_{5}\left(p_{4}\right)\left(p_{4}+p_{5}-m_{\Sigma}\right) k\left(p_{1}-p_{3}-m_{\Sigma}\right) p\left(p_{1}\right), \\
\mathcal{M}_{1 c}= & -2 e g_{K \Sigma \Xi_{5}} g_{K N \Sigma} \frac{1}{\left[\left(p_{2}-p_{4}\right)^{2}-m_{\Xi_{5}}^{2}\right]\left(t-m_{\Sigma}^{2}\right)} \\
& \times \bar{\Xi}_{5}\left(p_{4}\right) \xi\left(p_{4}-p_{2}+m_{\Xi_{5}}\right)\left(p_{1}-p_{3}-m_{\Sigma}\right) p\left(p_{1}\right),
\end{aligned}
$$




$$
\begin{aligned}
\mathcal{M}_{1 d}= & 2 e g_{K \Sigma \Xi_{5}} g_{K N \Sigma} \frac{1}{\left(s_{1}-m_{\Sigma}^{2}\right)\left(s-m_{N}^{2}\right)} \\
& \times \Xi\left(p_{4}\right)\left(p_{4}+p_{5}-m_{\Sigma}\right)\left(p_{1}+p_{2}+m_{N}\right) \\
& \times\left(k-\frac{\kappa_{p}}{2 m_{N}} k p_{2}\right) p\left(p_{1}\right), \\
\mathcal{M}_{1 e}= & 2 i g_{K \Sigma \Xi_{5}} g_{K^{*} N \Sigma} g_{\gamma K^{0} K^{* 0}} \frac{1}{\left(s_{1}-m_{\Sigma}^{2}\right)\left[\left(p_{2}-p_{3}\right)^{2}-m_{K^{*}}^{2}\right]} \\
& \times \epsilon_{\alpha \beta \mu \nu} p_{2}^{\alpha} p_{3}^{\beta} \epsilon^{\mu} \bar{\Xi}_{5}\left(p_{4}\right) \gamma_{5}\left(p_{4}+p_{5}+m_{\Sigma}\right) \gamma^{\nu} p\left(p_{1}\right),
\end{aligned}
$$

and those for the seven diagrams in Fig. 2 as

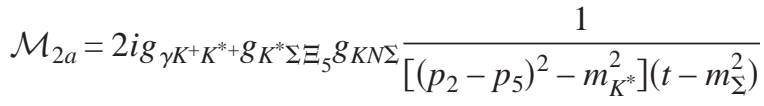

$$
\begin{aligned}
& \times \epsilon_{\alpha \beta \mu \nu} p_{2}^{\alpha} p_{5}^{\beta} \epsilon^{\mu} \bar{\Xi}_{5}\left(p_{4}\right) \gamma^{\nu}\left(p_{1}-p_{3}+m_{\Sigma}\right) \gamma_{5} n\left(p_{1}\right), \\
& \mathcal{M}_{2 b}=-4 e g_{K \Sigma \Xi_{5}} g_{K N \Sigma} \frac{1}{\left[\left(p_{2}-p_{5}\right)^{2}-m_{K}^{2}\right]\left(t-m_{\Sigma}^{2}\right)} \epsilon p_{5} \bar{\Xi}_{5}\left(p_{4}\right) \\
& \times\left(p_{1}-p_{3}-m_{\Sigma}\right) n\left(p_{1}\right), \\
& \mathcal{M}_{2 c}=2 e g_{K \Sigma \Xi_{5}} g_{K N \Sigma} \frac{1}{\left(s_{1}-m_{\Sigma}^{2}\right)\left(t-m_{\Sigma}^{2}\right)} \\
& \times \bar{\Xi}_{5}\left(p_{4}\right)\left(p_{4}+p_{5}-m_{\Sigma_{5}}\right) k\left(p_{1}-\not p_{3}-m_{\Sigma}\right) n\left(p_{1}\right), \\
& \mathcal{M}_{2 d}=4 e g_{K \Sigma \Xi_{5}} g_{K N \Sigma} \frac{1}{\left[\left(p_{2}-p_{4}\right)^{2}-m_{\Xi_{5}}^{2}\right]\left(t-m_{\Sigma}^{2}\right)} \\
& \times \bar{\Xi}_{5}\left(p_{4}\right) k\left(p_{4}-\not p_{2}+m_{\Xi_{5}}\right)\left(p_{1}-\not p_{3}-m_{\Sigma}\right) n\left(p_{1}\right), \\
& \mathcal{M}_{2 e}=-\frac{e \kappa_{n}}{m_{N}} g_{K \Sigma \Xi_{5}} g_{K N \Sigma} \frac{1}{\left(s_{1}-m_{\Sigma}^{2}\right)\left(s-m_{N}^{2}\right)} \bar{\Xi}_{5}\left(p_{4}\right)\left(p_{4}+p_{5}\right. \\
& \left.-m_{\Sigma}\right)\left(p_{1}+p_{2}+m_{N}\right) k p_{2} n\left(p_{1}\right) \text {, } \\
& \mathcal{M}_{2 f}=-4 e g_{K \Sigma \Xi_{5}} g_{K N \Sigma} \frac{1}{\left(s_{1}-m_{\Sigma}^{2}\right)\left[\left(p_{2}-p_{3}\right)^{2}-m_{K}^{2}\right]} \epsilon p_{3} \\
& \times \bar{\Xi}_{5}\left(p_{4}\right)\left(p_{4}+p_{5}-m_{\Sigma}\right) n\left(p_{1}\right) \text {, } \\
& \mathcal{M}_{2 g}=2 i g_{K \Sigma \Xi_{5}} g_{K^{*} N \Sigma} g_{\gamma K^{+} K^{*+}} \frac{1}{\left(s_{1}-m_{\Sigma}^{2}\right)\left[\left(p_{2}-p_{3}\right)^{2}-m_{K^{*}}^{2}\right]} \\
& \times \epsilon_{\alpha \beta \mu \nu} p_{2}^{\alpha} p_{3}^{\beta} \epsilon^{\mu} \bar{\Xi}_{5}\left(p_{4}\right) \gamma^{5}\left(p_{4}+p_{5}+m_{\Sigma}\right) \gamma^{\nu} n\left(p_{1}\right) \text {. }
\end{aligned}
$$

In the above, $p_{1}$ and $p_{2}$ denote, respectively, the momenta of nucleon and photon in the initial states; $p_{3}, p_{4}$, and $p_{5}$ are, respectively, those of the kaon on the left, the cascade, and the kaon on the right in the final states of the Feynman dia- grams in Figs. 1 and 2. We have also introduced the photon polarization vector $\epsilon^{\mu}$ and the definitions $s=\left(p_{1}+p_{2}\right)^{2}, t$ $=\left(p_{1}-p_{3}\right)^{2}$, and $s_{1}=\left(p_{4}+p_{5}\right)^{2}$.

\section{Form factors}

To take into account the effects due to hadron internal structure, a form factor is introduced to each amplitude, and it is taken to have the following form [40]:

$$
F\left(m_{x}, m_{y}\right)=\left[\frac{\Lambda^{4}}{\Lambda^{4}+\left(q_{x}^{2}-m_{x}^{2}\right)^{2}}\right]\left[\frac{\Lambda^{4}}{\Lambda^{4}+\left(q_{y}^{2}-m_{y}^{2}\right)^{2}}\right],
$$

where $q_{x}$ and $q_{y}$ are four-momenta of the intermediate offshell particles with masses $m_{x}$ and $m_{y}$ in each diagram, and $\Lambda$ is the cutoff parameter that characterizes the off-shell momentum above which hadron internal structure becomes important. Since the amplitudes for diagrams (1a) and (1e) in Fig. 1 for the reaction $\gamma p \rightarrow K^{0} K^{0} \Xi_{5}^{+}$are individually gauge invariant, including form factors does not affect their gauge invariance. This is different for the amplitudes for diagrams (1b)-(1d) as only their sum is gauge invariant. Using different form factors for these amplitudes thus leads to a violation of the gauge invariance. To recover the gauge invariance for the total amplitude, one can either drop the gauge violating terms in the amplitude [41] or use an averaged form factor [42] or other combinations of form factors [43] for these terms. For simplicity, we use here a common form factor, which is given by the average of the original form factors for diagrams (1b)-(1d), for all these three diagrams. A similar consideration is applied to the diagrams in Fig. 2 for the reaction $\gamma n \rightarrow K^{+} K^{+} \Xi_{5}^{--}$. In the following, we summarize the form factors used in the present study:

$$
\begin{gathered}
F_{(1 a)}=F_{(2 a)}=F\left(m_{\Sigma}, m_{K^{*}}\right), \\
F_{(1 b)}=F_{(1 c)}=F_{(1 d)}=\frac{1}{3}\left[F\left(m_{\Sigma}, m_{\Sigma}\right)+F\left(m_{\Sigma}, m_{\Xi_{5}}\right)\right. \\
\left.+F\left(m_{\Sigma}, m_{N}\right)\right], \\
F_{(1 e)}=F_{(2 g)}=F\left(m_{K^{*}}, m_{\Sigma}\right), \\
F_{(2 b)}=F_{(2 c)}=F_{(2 d)}=F_{(2 f)}=\frac{1}{4}\left[F\left(m_{\Sigma}, m_{K}\right)+F\left(m_{\Sigma}, m_{\Sigma}\right)\right. \\
\left.+F\left(m_{\Sigma}, m_{\Xi_{5}}\right)+F\left(m_{K}, m_{\Sigma}\right)\right], \\
F_{(2 e)}=F\left(m_{\Sigma}, m_{N}\right),
\end{gathered}
$$

where the subscripts refer to the diagrams in Figs. 1 and 2.

The value of the cutoff parameter is taken to be $\Lambda$ $=1.7 \mathrm{GeV}$, which is determined from fitting the measured cross section for charmed hadron production with three-body final states from photon-proton reactions at center-of-mass energy of $6 \mathrm{GeV}$ [44] using a similar hadronic model based on SU(4) flavor-invariant Lagrangians with empirical hadron masses and coupling constants. This cutoff parameter is larger than the value of $1.2 \mathrm{GeV}$ used in our previous study of the reaction $\gamma p \rightarrow K^{* 0} \Theta^{+}$[25], where the form factor was taken to be $F=\Lambda^{4} /\left(\Lambda^{4}+m_{x}^{4}\right)$ and the cutoff parameter was determined from fitting the cross section for charmed hadron 
production with two-body final states in photon-proton reactions.

\section{Cross sections for $\gamma p \rightarrow K^{0} K^{0} \Xi_{5}^{+}$and $\gamma n \rightarrow K^{+} K^{+} \Xi_{5}^{--}$}

As shown in the Appendix, the total cross section for these reactions can be expressed as

$$
\begin{aligned}
\sigma_{\gamma N \rightarrow K K \Xi_{5}}= & \frac{1}{(2 \pi)^{4}} \frac{1}{128 s p^{2}} \int_{s_{-}}^{s_{+}} d s_{1} \int_{t_{-}}^{t_{+}} d t \frac{k}{2 \sqrt{s_{1}}} \\
& \times \int_{0}^{\pi} \sin \theta d \theta \int_{0}^{\pi} d \phi\left|\sum_{i} \mathcal{M}_{i}\left(\gamma N \rightarrow K K \Xi_{5}\right)\right|^{2},
\end{aligned}
$$

where $s, t$, and $s_{1}$ were defined after Eq. (3). The limits of integrations are

$$
\begin{gathered}
s_{-}=\left(m_{\Xi_{5}}+m_{K}\right)^{2}, \quad s_{+}=\left(s^{1 / 2}-m_{K}\right)^{2}, \\
t_{ \pm}=m_{N}^{2}+m_{K}^{2} \\
-\frac{\left(m_{K}^{2}+s-s_{1}\right) \sqrt{m_{N}^{2}+p^{2}}}{\sqrt{s}} \pm 2 p \sqrt{\frac{\left(m_{K}^{2}+s-s_{1}\right)^{2}}{4 s}-m_{K}^{2}},
\end{gathered}
$$

where $p$ is the momentum of the nucleon in the total centerof-mass frame. We have also introduced by $\mathbf{k}$ the momentum of $\Xi_{5}$ in the center-of-mass frame of $\Xi_{5}$ and the kaon on the right of the Feynman diagram, with polar angles $\theta$ and $\phi$, i.e., the angles $\theta_{4}$ and $\phi_{4}$ shown in Fig. 5 of the Appendix.

\section{RESULTS}

We first show in the upper panels of Figs. 3 and 4 the cross sections for the reactions $\gamma p \rightarrow K^{0} K^{0} \Xi_{5}^{+}$and $\gamma n$ $\rightarrow K^{+} K^{+} \Xi_{5}^{--}$as functions of photon energy in the laboratory frame without including form factors at the strong interaction vertices. Both cross sections are seen to increase rapidly with increasing photon energy. Since the cross sections for the case of $g_{K^{*} \Sigma \Xi_{5}}=0$ are between those with $g_{K^{*} \Sigma \Xi_{5}}=1.27$ and $g_{K} \Sigma \Xi_{5}=-1.27$, the contribution from diagrams involving the coupling $g_{K^{*} \Sigma \Xi_{5}}$ is less important than those involving the coupling $g_{K \Sigma \Xi_{5}}$. In the lower panels, the cross sections with form factors are shown, and they are much smaller than those without form factors. Depending on the value of the coupling constant $g_{K^{*} \Sigma \Xi_{5}}$, the cross section for the reaction $\gamma p \rightarrow K^{0} K^{0} \Xi_{5}^{+}$has values of $0.03-0.6 \mathrm{nb}$ at $E_{\gamma}=4.5 \mathrm{GeV}$, while that for the reaction $\gamma n \rightarrow K^{+} K^{+} \Xi_{5}^{--}$has values of $0.1-0.6 \mathrm{nb}$. We note that these values are significantly smaller than those predicted for $\Theta^{+}$production in photonucleon reactions using the same hadronic model [23-25].

\section{SUMMARY}

Using a hadronic model that includes the coupling of pentaquark $\Xi_{5}$ to usual $\Sigma$ and $K$ or $K^{*}$, we have evaluated the cross sections for their production in the reactions $\gamma p$ $\rightarrow K^{0} K^{0} \Xi_{5}^{+}$and $\gamma n \rightarrow K^{+} K^{+} \Xi_{5}^{--}$by assuming that $\Xi_{5}$ has spin

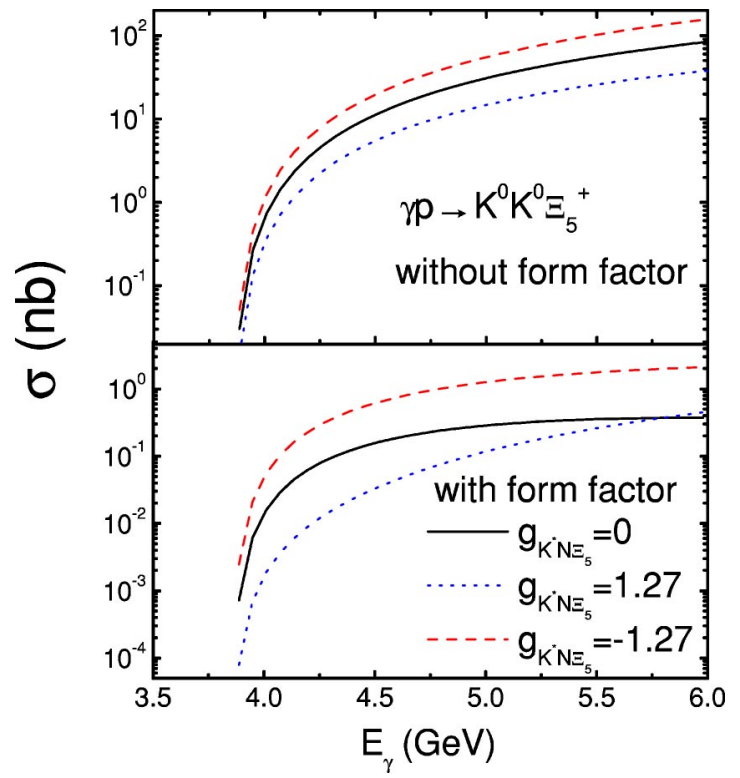

FIG. 3. (Color online) Total cross section for $\Xi_{5}^{+}$production from the reaction $\gamma p \rightarrow K^{0} K^{0} \Xi_{5}^{+}$as a function of photon energy and for the coupling constant $g_{K^{*} \Sigma \Xi_{5}}=1.27$ (dotted curve), 0 (solid curve), and -1.27 (dashed curve). Upper and lower panels are for the cases without and with form factors, respectively.

$1 / 2$ and positive parity. Using coupling constants related to those for pentaquark $\Theta^{+}$couplings to $N$ and $K$ or $K^{*}$, and also including form factors at the strong interaction vertices with empirical cutoff parameters, these cross sections are found in the range of $0.03-0.6 \mathrm{nb}$ for $\gamma p \rightarrow K^{0} K^{0} \Xi_{5}^{+}$and $0.1-0.6 \mathrm{nb}$ for $\gamma n \rightarrow K^{+} K^{+} \Xi_{5}^{--}$at photon energy $E_{\gamma}=4.5 \mathrm{GeV}$. Since the coupling constant $g_{K N \Theta}$ is much smaller for a negative parity

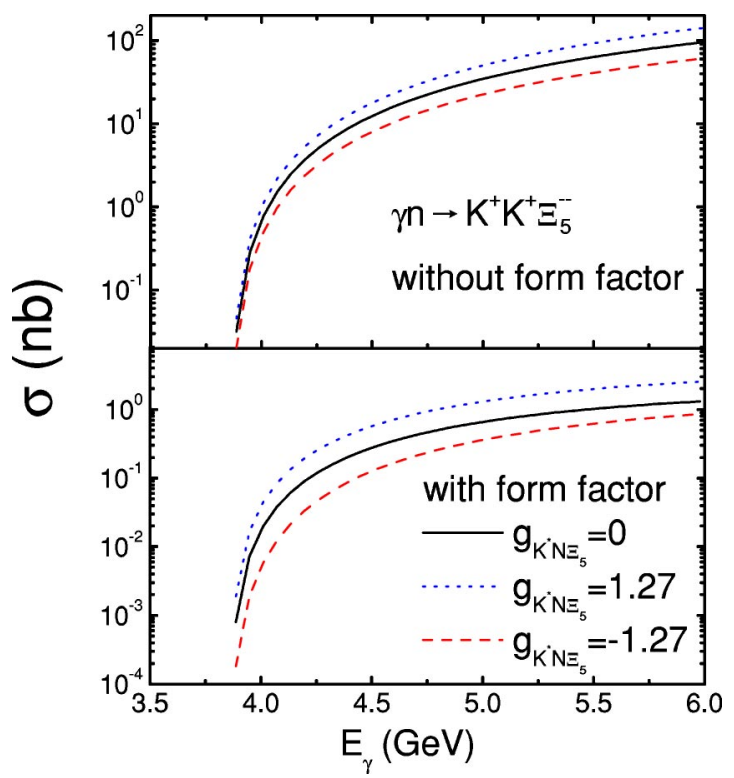

FIG. 4. (Color online) Total cross section for $\Xi_{5}^{--}$production from the reaction $\gamma n \rightarrow K^{+} K^{+} \Xi_{5}^{--}$as a function of photon energy and for the coupling constant $g_{K^{*} \Sigma \Xi_{5}}=1.27$ (dotted curve), 0 (solid curve), and -1.27 (dashed curve). Upper and lower panels are for the case without and with form factors, respectively. 
$\Theta^{+}$than for a positive parity one, the coupling constant $g_{K \Sigma \Xi_{5}}$ also becomes smaller if $\Xi_{5}$ has a negative parity. As a result, the cross sections for producing negative pentaquark $\Xi_{5}^{+}$and $\Xi_{5}^{--}$are much smaller than for positive ones as in the case of $\Theta^{+}$production. Although the cross sections for photoproduction of pentaquark $\Xi_{5}$ are an order of magnitude smaller than those for producing the $\Theta^{+}$in these reactions, study of $\Xi_{5}$ production will be useful for understanding the properties of pentaquark baryons.

\section{ACKNOWLEDGMENTS}

This paper was based on work supported in part by the U.S. National Science Foundation under Grant No. PHY0098805 and the Welch Foundation under Grant No. A-1358.

\section{APPENDIX}

In this appendix, we describe the derivation of the cross section formula shown in Eq. (6) for the reactions $\gamma p$ $\rightarrow K^{0} K^{0} \Xi_{5}^{+}$and $\gamma n \rightarrow K^{+} K^{+} \Xi_{5}^{--}$involving two particles in the initial state and three particles in the final state, i.e., $1+2$ $\rightarrow 3+4+5$. It essentially follows the method given in Ref. [45].

In the center-of-mass frame of particles 1 and 2, the cross section for such a reaction generally reads as

$$
\begin{aligned}
\sigma_{1+2 \rightarrow 3+4+5}= & \frac{1}{(2 \pi)^{5}} \frac{1}{4 s^{1 / 2} p} \iint d^{4} p_{3} d^{4} p_{\Delta} \delta\left(p_{3}^{2}-m_{3}^{2}\right) \\
& \times \delta^{(4)}\left(p_{\Delta}-p_{1}+p_{3}\right) \iint d^{4} p_{4} d^{4} p_{5} \delta\left(p_{4}^{2}-m_{4}^{2}\right) \\
& \times \delta\left(p_{5}^{2}-m_{5}^{2}\right) \delta^{(4)}\left(q-p_{4}-p_{5}\right) \\
& \times\left|\mathcal{M}\left(p_{1}+p_{2} \rightarrow p_{3}+p_{4}+p_{5}\right)\right|^{2}
\end{aligned}
$$

with $s=\left(p_{1}+p_{2}\right)^{2}, q=p_{1}+p_{2}-p_{3}$, and $p$ denoting the momentum of initial particles in their center-of-mass frame.

To evaluate the phase-space integral in Eq. (A1), we choose the coordinate system in the center-of-mass frame of particles 4 and 5 as shown in Fig. 5, in which the $z$ axis is along the three-momentum $\mathbf{p}_{2}$ of particle 2 , while $\mathbf{p}_{1}$ and $\mathbf{p}_{3}$ are in the $x-z$ plane. In terms of the angles shown in the figure, the four-momenta of these particles can be expressed as

$$
\begin{gathered}
p_{1}=\left(E_{1},\left|\mathbf{p}_{3}\right| \sin \theta_{3}, 0,\left|\mathbf{p}_{3}\right| \cos \theta_{3}-\left|\mathbf{p}_{2}\right|\right), \\
p_{2}=\left(E_{2}, 0,0,\left|\mathbf{p}_{2}\right|\right) \\
p_{3}=\left(E_{3},\left|\mathbf{p}_{3}\right| \sin \theta_{3}, 0,\left|\mathbf{p}_{3}\right| \cos \theta_{3}\right)
\end{gathered}
$$

$p_{4}=\left(E_{4},\left|\mathbf{p}_{4}\right| \sin \theta_{4} \sin \phi_{4},\left|\mathbf{p}_{4}\right| \sin \theta_{4} \cos \phi_{4},\left|\mathbf{p}_{4}\right| \cos \theta_{4}\right)$,

$$
\begin{aligned}
p_{5}= & \left(E_{5},-\left|\mathbf{p}_{4}\right| \sin \theta_{4} \sin \phi_{4},-\left|\mathbf{p}_{4}\right| \sin \theta_{4} \cos \phi_{4},\right. \\
& \left.-\left|\mathbf{p}_{4}\right| \cos \theta_{4}\right) .
\end{aligned}
$$

From four-momentum conservation and on-shell constraints, the following identities can be derived:

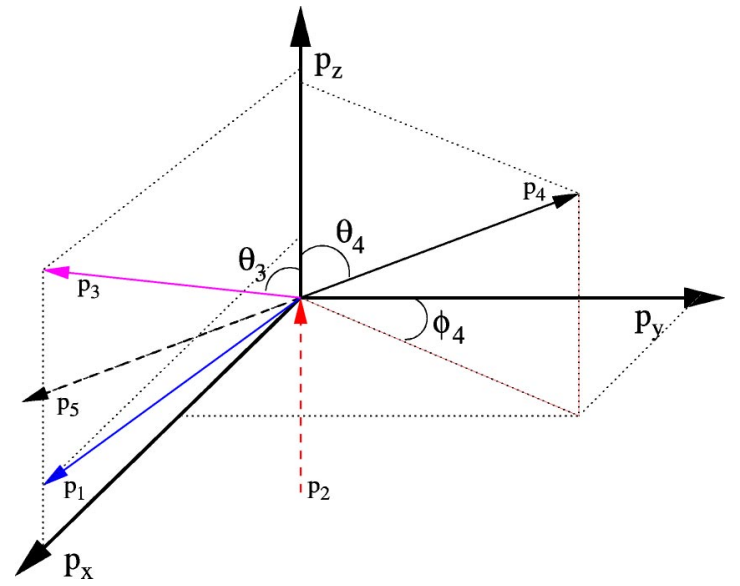

FIG. 5. (Color online) Coordinate system used for the threemomenta of the particles in the reaction $1+2 \rightarrow 3+4+5$.

$$
\begin{gathered}
E_{1}=\frac{t+s-m_{2}^{2}-m_{3}^{2}}{2 \sqrt{s_{1}}}, \quad E_{2}=\frac{s_{1}-t+m_{2}^{2}}{2 \sqrt{s_{1}}}, \quad E_{3}=\frac{s-s_{1}-m_{3}^{2}}{2 \sqrt{s_{1}}}, \\
E_{4}=\frac{s_{1}+m_{4}^{2}-m_{5}^{2}}{2 \sqrt{s_{1}}}, \quad E_{5}=\frac{s_{1}+m_{5}^{2}-m_{4}^{2}}{2 \sqrt{s_{1}}}, \\
\cos \theta_{3}=\frac{m_{1}^{2}-E_{1}^{2}+\left|\mathbf{p}_{3}\right|^{2}+\left|\mathbf{p}_{2}\right|^{2}}{2\left|\mathbf{p}_{3}\right|\left|\mathbf{p}_{2}\right|}, \\
\left|\mathbf{p}_{2}\right|=\sqrt{E_{2}^{2}-m_{2}^{2}}, \quad\left|\mathbf{p}_{3}\right|=\sqrt{E_{3}^{2}-m_{3}^{2}}, \quad\left|\mathbf{p}_{4}\right|=\sqrt{E_{4}^{2}-m_{4}^{2}},
\end{gathered}
$$

where $t=\left(p_{1}-p_{3}\right)^{2}$ and $s_{1}=\left(p_{4}+p_{5}\right)^{2}$. The two-body phasespace integral can be rewritten as

$$
\begin{gathered}
\int d^{4} p_{4} d^{4} p_{5} \delta\left(p_{4}^{2}-m_{4}^{2}\right) \delta\left(p_{5}^{2}-m_{5}^{2}\right) \delta^{(4)}\left(q-p_{4}-p_{5}\right) \\
=\frac{k}{2 \sqrt{s_{1}}} \int_{0}^{\pi} \sin \theta_{4} d \theta_{4} \int_{0}^{\pi} d \phi_{4},
\end{gathered}
$$

where $k$ is the momentum of particle 4 or 5 in their centerof-mass frame.

After integrating over the four-momentum $p_{3}$, we have

$$
\begin{aligned}
\sigma_{1+2 \rightarrow 3+4+5}= & \frac{1}{(2 \pi)^{5}} \frac{1}{4 s^{1 / 2} p} \int d^{4} p_{\Delta} \delta\left(\left(p_{1}-p_{\Delta}\right)^{2}-m_{3}^{2}\right) \\
& \times \frac{k}{2 \sqrt{s_{1}}} \int_{0}^{\pi} \sin \theta_{4} d \theta_{4} \int_{0}^{\pi} d \phi_{4} \mid \mathcal{M}\left(p_{1}+p_{2} \rightarrow p_{3}\right. \\
& \left.+p_{4}+p_{5}\right)\left.\right|^{2} .
\end{aligned}
$$


Denoting $p_{\Delta}=\left(E_{\Delta}, \mathbf{p}_{\Delta}\right)$, and then using the identities

$$
\begin{gathered}
s_{1}=m_{3}^{2}-m_{1}^{2}+m_{2}^{2}+2 \sqrt{s} E_{\Delta}, \\
t \equiv p_{\Delta}^{2}=m_{3}^{2}-m_{1}^{2}+2 \sqrt{m_{1}^{2}+p^{2}} E_{\Delta}-2 p p_{\Delta z}
\end{gathered}
$$

in the center-of-mass frame of particles 1 and 2, we can rewrite

$$
\int d^{4} p_{\Delta} \delta\left(\left(p_{1}-p_{\Delta}\right)^{2}-m_{3}^{2}\right)=\frac{\pi}{4 p s^{1 / 2}} \int d s_{1} \int d t
$$

We then have

$$
\begin{aligned}
\sigma_{1+2 \rightarrow 3+4+5}= & \frac{1}{(2 \pi)^{4}} \frac{1}{32 s p^{2}} \int_{s_{-}}^{s_{+}} d s_{1} \int_{t_{-}}^{t_{+}} d t \\
& \times \frac{k}{2 \sqrt{s_{1}}} \int_{0}^{\pi} \sin \theta_{4} d \theta_{4} \int_{0}^{\pi} d \phi_{4} \mid \mathcal{M}\left(p_{1}+p_{2} \rightarrow p_{3}\right. \\
& \left.+p_{4}+p_{5}\right)\left.\right|^{2},
\end{aligned}
$$

where

$$
\begin{gathered}
s_{-}=\left(m_{4}+m_{5}\right)^{2}, s_{+}=\left(s^{1 / 2}-m_{3}\right)^{2}, \\
t_{ \pm}=m_{1}^{2}+m_{3}^{2} \\
-\frac{\left(m_{3}^{2}+s-s_{1}\right) \sqrt{m_{1}^{2}+p^{2}}}{\sqrt{s}} \pm 2 p \sqrt{\frac{\left(m_{3}^{2}+s-s_{1}\right)^{2}}{4 s}-m_{3}^{2} .}
\end{gathered}
$$

[1] D. Diakonov, V. Petrov, and M. Polyakov, Z. Phys. A 359, 305 (1997).

[2] T. Nakano et al., Phys. Rev. Lett. 91, 012002 (2003).

[3] S. Stepanyan et al., CLAS Collaboration, hep-ex/0307018.

[4] V. Kubarovsky and S. Stepanyan, hep-ex/0307088.

[5] J. Barth et al., SAPHIR Collaboration, Phys. Lett. B 572, 127 (2003).

[6] V. V. Barmin et al., Phys. At. Nucl. 66, 1715 (2003).

[7] M. Praszalowicz, Phys. Lett. B 575, 234 (2003).

[8] M. V. Polyakov and A. Rathke, Eur. Phys. J. A 18, 691 (2003).

[9] H. Walliser and V. B. Kopeliovich, J. Exp. Theor. Phys. 97, 433 (2003).

[10] B. K. Jennings and K. Maltman, hep-ph/0308286.

[11] D. Borisyuk, M. Faber, and A. Kobushkin, hep-ph/0307370.

[12] N. Itzhaki, I. R. Klebanov, P. Ouyang, and L. Rastelli, Nucl. Phys. B684, 264 (2004).

[13] Fl. Stancu and D. O. Riska, Phys. Lett. B 575, 242 (2003).

[14] M. Karliner and H. J. Lipkin, hep-ph/0307243.

[15] R. L. Jaffe and F. Wilczek, Phys. Rev. Lett. 91, 232003 (2003).

[16] A. Hosaka, Phys. Lett. B 571, 55 (2003).

[17] L. Y. Glozman, Phys. Lett. B 575, 18 (2003).

[18] S. L. Zhu, Phys. Rev. Lett. 91, 232002 (2003).

[19] R. D. Matheus, F. S. Navarra, M. Nielsen, R. Rodrigues da Silva, and S. H. Lee, Phys. Lett. B 578, 323 (2004).

[20] J. Sugiyama, T. Doi, and M. Oka, Phys. Lett. B 581, 167 (2004).

[21] S. Sasaki, hep-lat/0310014.

[22] F. Ciskor, Z. Fodor, S. D. Katz, and T. G. Kovács, J. High Energy Phys. 11, 070 (2003).

[23] W. Liu and C. M. Ko, Phys. Rev. C 68, 045203 (2003).

[24] W. Liu and C. M. Ko, nucl-th/0309023.

[25] W. Liu, C. M. Ko, and V. Kubarovsky, Phys. Rev. C 69, 025202 (2004).
[26] Y. Oh, H. Kim, and S. H. Lee, Phys. Rev. D 69, 014009 (2004); hep-ph/0311054.

[27] S. I. Nam, A. Hosaka, and H. C. Kim, Phys. Lett. B 579, 43 (2004).

[28] Q. Zhao, Phys. Rev. D 69, 053009 (2004).

[29] T. Hyodo, A. Hosaka, and E. Oset, Phys. Lett. B (to be published), nucl-th/0307105.

[30] K. Nakayama and K. Tsushima, Phys. Lett. B 583, 269 (2004).

[31] C. E. Garlson, C. D. Carone, H. J. Kwee, and V. Nazaaryan, Phys. Lett. B 573, 101 (2003).

[32] X. Chen, Y. Mao, and B. Q. Ma, hep-ph/0307381.

[33] C. Alt et al. NA49 Collaboration, Phys. Rev. Lett. 92, 042003 (2004).

[34] C. H. Li and C. M. Ko, Nucl. Phys. A712, 110 (2002).

[35] B. Holzenkamp, K. Holinde, and J. Speth, Nucl. Phys. A500, 485 (1989).

[36] G. Janssen, K. Holinde, and J. Speth, Phys. Rev. C 54, 2218 (1996).

[37] R. A. Adelseck and B. Saghai, Phys. Rev. C 42, 108 (1990).

[38] Y. Oh, H. Kim, and S. H. Lee, hep-ph/0310117.

[39] K. Hagiwara et al., Particle Data Group, Phys. Rev. D 66, 010001 (2002).

[40] W. S. Chung, G. Q. Li, and C. M. Ko, Phys. Lett. B 401, 1 (1997).

[41] K. Ohta, Phys. Rev. C 40, 1335 (1989).

[42] H. Haberzettl, C. Bennhold, T. Mart, and T. Feuster, Phys. Rev. C 58, R40 (1998).

[43] R. M. Davidson and R. Workman, Phys. Rev. C 63, 058201 (2001).

[44] W. Liu, S. H. Lee, and C. M. Ko, Nucl. Phys. A724, 375 (2003).

[45] W. Beenakker, H. Kuijf, W. L. van Neerven, and J. Smith, Phys. Rev. D 40, 54 (1989). 